Commun. math. Phys. 24, 289-302 (1972)

(C) by Springer-Verlag 1972

\title{
Einstein's Equations and Locality
}

\author{
L. BRACCI \\ Istituto di Fisica dell'Università di Pisa \\ and \\ Scuola Normale Superiore, Pisa, Italy \\ F. STROCCHI ${ }^{\star}$ \\ Department of Physics, University of Princeton, Princeton, N. J., USA
}

Received May, 27/October 25, 1971

\begin{abstract}
The problem of formulating a local quantum theory of Einstein equations is examined. It is proved that Einstein equations cannot hold as operator equations if written in terms of a potential $h_{\mu \nu}(x)$ which is a weakly local field. This result is independent of the kind of metric chosen in the Hilbert space and it doesn't require covariance of $h_{\mu v}$.

As a consequence, the peculiar features of the radiation gauge method, i.e. non locality and non covariance, appear as necessary features of any solution not involving unphysical particles.
\end{abstract}

\section{Introduction}

The object of the present paper is to analyse those difficulties [1] which arise in the quantization of the Einstein equations, mainly because of the zero mass of the gravitons. This kind of difficulties are strictly connected with the introduction of the potentials, i.e. with the gauge problem, and have a counterpart in the simpler case of quantum electrodynamics [2]. In the theory of gravitation, however, they seem to have deeper implications because the "potentials" $g_{\mu \nu}$ are strictly related to the geometry of the four-dimensional space.

Wightman's approach to quantum field theory appears as the natural framework to deal with this problem and his philosophy will be adopted throughout the paper. When discussed in the language of axiomatic field theory, the problem of quantizing the Einstein equation shows his basic difficulties in a clear way. As it will be shown, they have very little to do with subsidiary conditions, indefinite metric etc. as usually stated in the literature. On the contrary, they are strictly connected with two basic assumptions of quantum field theory: Lorentz covariance and locality.

* On leave of absence from: Istituto Nazionale di Fisica Nucleare, Sezione di Pisa, Pisa, Italy. 
More precisely, one cannot quantize the Einstein equations in terms of a potential $h_{\mu \nu}(x)$ which is a covariant and/or weakly local field. As a matter of fact, we will prove that the Einstein equations cannot even hold on the vacuum state

$$
R_{\mu \nu} \Psi_{0}=0 \quad \varepsilon^{\mu \nu \varrho \sigma} \partial_{v} R_{\lambda \tau \varrho \sigma} \Psi_{0}=0
$$

in a Hilbert space in which the potential $h_{\mu \nu}(x)$ is defined as a covariant and/or weakly local operator valued distribution. Otherwise, one would get that all the Wightman functions of the field $R_{\mu \nu \rho \sigma}$ vanish and the theory is trivial. This result is obtained independently of the metric in the Hilbert space.

As a consequence, the peculiar features of the radiation gauge method [3], i.e. non locality and non covariance, appear as necessary features of any solution not involving unphysical particles.

\section{Quantization of the Einstein's Equations in the Linear Approximation. Basic Assumptions}

In order to simplify the discussion, we will consider the Einstein's equations in vacuum, in the weak-field approximation. Clearly, the difficulties we will find in this case will be present also in the more general case. In fact, if a complete theory exists, it must make sense also in the weak-field approximation. This situation is described by the following equations [4]

where

$$
R_{\mu v}(x)=0 \quad R(x)=0
$$

Here $g^{(0) \lambda \varrho}$ stands for the constant metric tensor, $g^{(0) 00}=1, g^{(0) i i}=-1$, $g^{(0) \lambda \varrho}=0$ for $\lambda \neq \varrho$. For simplicity, in the following we will omit the upper label ${ }^{(0)}$. Besides Eqs. (1) and (2), the gravitational field $R_{\mu \nu \varrho \sigma}$ satisfies the following identities [4]

$$
\begin{aligned}
& R_{\lambda \mu v \varrho}=-R_{\mu \lambda v \varrho}=R_{\mu \lambda \varrho v}=R_{v \varrho \lambda \mu}, \\
& R_{\lambda v \varrho \sigma}+R_{\lambda \sigma v \varrho}+R_{\lambda \varrho \sigma v}=0, \\
& \varepsilon^{\mu \nu \varrho \sigma} \partial_{v} R_{\alpha \beta \varrho \sigma}=0 \quad \text { (Bianchi's identities). }
\end{aligned}
$$

The set of Eqs. (3)-(5) imply that the basic fields $R_{\mu \nu \rho \sigma}(x)$ may be written in terms of lower order tensors $h_{\mu v}(x)$ in the following way

$$
R_{\mu \nu \varrho \sigma}(x)=\frac{1}{2}\left(\partial_{v} \partial_{\varrho} \delta_{\mu}^{\alpha} \delta_{\sigma}^{\beta}+\partial_{\mu} \partial_{\sigma} \delta_{v}^{\alpha} \delta_{\varrho}^{\beta}-\partial_{v} \partial_{\sigma} \delta_{\varrho}^{\alpha} \delta_{\mu}^{\beta}-\partial_{\varrho} \partial_{\mu} \delta_{v}^{\alpha} \delta_{\sigma}^{\beta}\right) h_{\alpha \beta}(x)
$$

where $h_{\mu \nu}(x)=h_{v \mu}(x)$ are defined as operator valued distributions in $\mathscr{H}$. 
Therefore, the quantization of $R_{\mu \nu \varrho \sigma}(x)$ is reduced to the problem of quantizing the fields $h_{\mu \nu}(x)$. The need of using the quantities $h_{\mu \nu}(x)$ in a quantum field theory of gravitation follows also from the fact that $h_{\mu v}(x)\left(\right.$ not $R_{\mu v \varrho \sigma}$ ) enter in any local interaction ${ }^{1}$ and in $S$-matrix elements and that by using only the fields $R_{\mu \nu \varrho \sigma}(x)$ one cannot account for the production and absorption of soft gravitons (long range forces) [5]. In addition the field $h_{\mu v}(x)$ are strictly related to the metric of the four dimensional space and play a fundamental role in the theory of gravitation.

In this section we will consider the basic properties a quantum field theory of Einstein's equations must have. In a certain sense the following basic "assumptions" may be regarded as a definition of our problem [6]:

a) The fields $h_{\mu \nu}(x), \mu, v=0,1,2,3$, may be defined as operator valued distributions [7] in a Hilbert space $\mathscr{H}$.

b) There exists a "unitary" ${ }^{2}$ representation of the Poincaré group $\{a, \Lambda\} \rightarrow U(a, \Lambda)$ such that the fields $R_{\mu \nu \rho \sigma}(x)$ transform as tensor fields under $U(a, \Lambda)$

$$
U(a, \Lambda) R_{\mu \nu \varrho \sigma}(x) U(a, \Lambda)^{-1}=\Lambda_{\mu}^{-1 \alpha} \Lambda_{\nu}^{-1 \beta} \Lambda_{\varrho}^{-1 \gamma} \Lambda_{\sigma}^{-1 \delta} R_{\alpha \beta \gamma \delta}(\Lambda x+a)
$$

and the fields $h_{\mu v}(x)$ have the following transformation properties under the space time translation group

$$
U(a, 1) h_{\mu \nu}(x) U(a, 1)^{-1}=h_{\mu \nu}(x+a) .
$$

c) There exists a state $\Psi_{0}$ (vacuum state) which is invariant under $U(a, \Lambda)$

$$
U(a, \Lambda) \Psi_{0}=\Psi_{0}
$$

and the spectral condition is satisfied by the generators of $U(a, 1)^{3}$.

It is important to stress that no assumption has been made about the transformation properties of $h_{\mu \nu}$ under the Lorentz group ${ }^{4}$. As a matter of fact $h_{\mu \nu}(x)$ are not observable quantities and there is no need for requiring that $h_{\mu \nu}(x)$ transform as the components of a tensor field. One may, however, show that the transformation properties of $h_{\mu v}(x)$ under $U(0, \Lambda)$ are not arbitrary as a consequence of condition (7).

${ }^{1}$ As a matter of fact, it seems that one cannot write a local interaction Lagrangian involving $R_{\mu \nu \rho \sigma}(x)$.

${ }^{2}$ Unitarity is here defined in terms of the metric of $\mathscr{H}$. It may be that all the physically meaningful quantities have to be defined in terms of "products" which do not coincide with the ordinary scalar product in $\mathscr{H}$. In this case unitarity is defined in terms of such new "products".

${ }^{3}$ It is worthwhile to note that conditions a), b) and c) are obviously satisfied in the standard quantizations of the Einstein equations like the Gupta formulation or the radiation gauge method, in spite of the many contradictory statements one may find in the literature.

${ }^{4}$ Even if we shall not assume all of the Wightman's axioms, we shall use the Wightman formulation as guide. 
Statement 1. If $R_{\mu \nu \varrho \sigma}(x)$ transform according to Eq. (7), the fields $h_{\mu \nu}(x)$ transform in the following way under the Lorentz group:

$\Lambda_{\mu}^{-1 \varrho} \Lambda_{v}^{-1 \sigma} U(0, \Lambda)^{-1} h_{\varrho \sigma}(\Lambda x) U(0, \Lambda)=h_{\mu \nu}(x)+\partial_{\mu} \mathscr{F}_{\nu}(x ; \Lambda)+\partial_{\nu} \mathscr{F}_{\mu}(x ; \Lambda)$

where the "fields" $\mathscr{F}_{v}(x ; \Lambda)$ do not necessarily transform as tensors under $U(0, \Lambda)$.

Proof. Without loss of generality we define

$$
\mathscr{F}_{\mu v}(x ; \Lambda) \equiv \Lambda_{\mu}^{-1 \varrho} \Lambda_{\nu}^{-1 \sigma} U(0, \Lambda)^{-1} h_{\varrho \sigma}(\Lambda x) U(0, \Lambda)-h_{\mu v}(x)=\mathscr{F}_{\nu \mu}(x ; \Lambda)
$$

By using Eq. (6), and Eq. (7) it is not difficult to see that $\mathscr{F}_{\mu \nu}(x ; \Lambda)$ must satisfy the following equation

$$
\partial_{\mu} \partial_{\nu} \mathscr{F}_{\lambda \varrho}(x ; \Lambda)+\partial_{\lambda} \partial_{\varrho} \mathscr{F}_{\mu \nu}(x ; \Lambda)-\partial_{\lambda} \partial_{\nu} \mathscr{F}_{\varrho \mu}(x ; \Lambda)-\partial_{\varrho} \partial_{\mu} \mathscr{F}_{\lambda \nu}(x ; \Lambda)=0 .
$$

The above equation implies that $\mathscr{F}_{\mu \nu}$ is a "gauge" field and therefore it must have the following form

$$
\mathscr{F}_{\mu \nu}(x ; \Lambda)=\partial_{\mu} \mathscr{F}_{\nu}(x ; \Lambda)+\partial_{\nu} \mathscr{F}_{\mu}(x ; \Lambda) .
$$

In concluding this section we want to stress that no assumption has been made about the metric in the Hilbert space $\mathscr{H}$, in which $h_{\mu \nu}$ are defined as operator valued distributions. The "product" of two vectors $\Psi_{1}$, $\Psi_{2}$, may be defined as a sesquilinear form

$$
\left(\Psi_{1}, \Psi_{2}\right)=\left\langle\eta \Psi_{1}, \Psi_{2}\right\rangle
$$

where $\langle$,$\rangle is the scalar product in \mathscr{H}$ and $\eta$ is the metric operator which may be non-positive definite. It may be that the physically meaningful quantities like vacuum expectation values, transition probabilities etc., have to be defined in terms of the products $($, ) instead of the products $\langle$,$\rangle . This is the case usually referred to as indefinite metric [9]. It is$ worthwhile to remark that in this case the operators $U(a, \Lambda)$ must be unitary with respect to the product $($,$) :$

$$
U^{+}=\eta U^{-1} \eta
$$

The following results do not depend on whether $\eta$ is a positive definite operator or not.

\section{Quantization of Einstein's Equations and Lorentz Covariance}

In this section we shall discuss the implications of the assumption of Lorentz covariance in the quantum field theory of Einstein's equations. A natural question is whether one may assume that the fields $h_{\mu \nu}(x)$ transform as the components of a second rank tensor under the Lorentz group

$$
U(0, \Lambda) h_{\mu \nu}(x) U(0, \Lambda)^{-1}=\Lambda_{\mu}^{-1 \varrho} \Lambda_{\nu}^{-1 \sigma} h_{\varrho \sigma}(\Lambda x)
$$


This is equivalent to put $\mathscr{F}_{\mu}(x ; \Lambda)=0$ in Eq. (9).

As discussed in detail in Ref. [10], one may prove that the addition of condition $b^{\prime \prime}$ ) to the properties $a^{\prime}$ ), $b^{\prime}$ ), c) and d), leads to a trivial theory, i.e. one has

$$
\left(\Psi_{0}, R_{\lambda \mu \nu \varrho}(x) R_{\alpha \beta \gamma \delta}(y) \Psi_{0}\right)=0 .
$$

For the details of the proof and a discussion of the results we refer the reader to Ref. [10].

Therefore, a quantum field theory of Einstein's equations requires the use of a gravitational potential $h_{\mu \nu}(x)$ which does not transform covariantly under the Lorentz group. Thus, the use of non covariant fields, which is in general regarded as a feature of the radiation gauge method [11] is an unavoidable step in the quantization of Einstein's equations, as operator equations satisfied on the physical states.

Finally, we want to remark that the above results prove that one cannot define spin two projection operators in contrast to what is sometimes stated in the literature [12]. As a matter of fact, if one could give any meaning to operators like

$$
\begin{aligned}
P^{(2)}= & \frac{\left(\hat{S}^{2}-2\right) \hat{S}^{2}}{24} \\
= & \frac{1}{6}\left\{3 \delta_{\mu_{1} v_{1}} \delta_{\mu_{2} v_{2}}+3 \delta_{\mu_{1} v_{2}} \delta_{\mu_{2} v_{1}}-2 \delta_{\mu_{1} \mu_{2}} \delta_{v_{1} v_{2}}+\frac{4}{\square^{2}} \partial_{\mu_{1}} \partial_{\mu_{2}} \partial_{v_{1}} \partial_{v_{2}}\right. \\
& -\frac{3}{\square}\left[\delta_{\mu_{1} v_{1}} \partial_{\mu_{2}} \partial_{v_{2}}+\delta_{\mu_{2} v_{2}} \partial_{\mu_{1}} \partial_{v_{1}}+\delta_{\mu_{1} v_{2}} \partial_{\mu_{2}} \partial_{v_{1}}+\delta_{\mu_{2} v_{1}} \partial_{\mu_{1}} \partial_{v_{2}}\right. \\
& \left.\left.-\frac{2}{3}\left(\delta_{\mu_{1} \mu_{2}} \partial_{v_{1}} \partial_{v_{2}}+\delta_{v_{1} v_{2}} \partial_{\mu_{1}} \partial_{\mu_{2}}\right)\right]\right\}
\end{aligned}
$$

which are ill-defined in the case of massless fields, one would obtain fields

$$
h_{\mu \nu}^{(2)}(x) \equiv P_{\mu \nu}^{(2) \lambda \varrho} h_{\lambda \varrho}(x)
$$

which satisfy the following equations

$$
\begin{gathered}
\square h_{\mu \nu}^{(2)}(x)=0, \\
\partial^{\mu}\left(h_{\mu \nu}^{(2)}-\frac{1}{2} g_{\mu \nu} h_{\lambda}^{(2) \lambda}\right)=0 .
\end{gathered}
$$

The above Eqs. (13), (13') are equivalent to the Einstein's equations. Thus, one would get a theory to which the above results apply, i.e.

$$
\left(\Psi_{0}, R_{\lambda \mu \nu \varrho}(x) R_{\alpha \beta \gamma \delta}(y) \Psi_{0}\right)=0
$$

and consequently [10]

$$
h_{\mu \nu}^{(2)}=\partial_{\mu} h_{v}+\partial_{v} h_{\mu}
$$

This contradicts the definition of the projection operator $P$, according to which $h_{\mu \nu}^{(2)}$ should be the spin-two part of the field $h_{\mu v}$. 


\section{Quantization of Einstein's Equations and Weak Local Commutativity}

Another basic property one would like to have for the fields $h_{\mu v}(x)$ is microscopic causality or weak local commutativity (WLC). Thus, in the present section we shall investigate the possibility of constructing a local quantum theory of Einstein's equations by using non covariant potentials $h_{\mu v}(x)$ which are weakly local fields.

Again we will find that, like Lorentz covariance, WLC cannot be required for the fields $h_{\mu v}(x)$. Otherwise, one would get a trivial theory.

Microscopic causality or weak local commutativity means that the fields $h_{\mu \nu}$ satisfy the following condition

e) $\quad\left(\Psi_{0},\left[h_{\mu v}\left(f^{\mu v}\right), h_{\varrho \sigma}\left(g^{\varrho \sigma}\right)\right] \Psi_{0}\right)=0$

if the support of the test function $f^{\mu \nu}$ is spacelike with respect to the support of $g^{\rho \sigma}$. Here and in the following the operators $h_{\mu v}\left(f^{\mu \nu}\right)$ are defined in the following way

$$
h_{\mu v}\left(f^{\mu \nu}\right) \equiv h(f)=\sum_{\mu, v} \int h_{\mu \nu}(x) f^{\mu \nu}(x) d^{4} x .
$$

In terms of Wightman function WLC is expressed in the following way:

$$
W_{\mu \nu \varrho \sigma}(x-y)=W_{\varrho \sigma \mu \nu}(y-x) \text { if }(x-y)^{2}<0 .
$$

Weak local commutativity constitutes the simplest and most direct way of imposing causality in the theory of quantized fields. Furthermore, it is a fundamental requirement in order to prove some general theorems of quantum field theory, like the PCT theorem or the theorem on the connection between spin and statistics [13]. In order to give a meaning to condition e) one has to require that the operator valued distribution $h_{\mu v}(x)$ can be smeared with test functions with compact support. For example the class of strictly local fields satisfy this requirement [8].

\section{Impossibility of a Weakly Local Quantum Theory of Einstein's Equations}

In this section we will give the details of the proof that $h_{\mu \nu}(x)$ cannot be a weakly local field. To this purpose we consider the two-point function

$$
W_{\mu \nu \alpha \beta \gamma \delta}(x-y)=\left(\Psi_{0}, h_{\mu v}(x) R_{\alpha \beta \gamma \delta}(y) \Psi_{0}\right)
$$


Lemma 1. The two point function $W_{\mu \nu \alpha \beta \gamma \delta}(x)$ transforms covariantly under the homogeneous Lorentz group, i.e.

$$
W_{\mu \nu \alpha \beta \gamma \delta}(\Lambda x)=\Lambda_{\mu}^{\lambda} \Lambda_{v}^{e} \Lambda_{\alpha}^{\varepsilon} \Lambda_{\beta}^{\zeta} \Lambda_{\gamma}^{\eta} \Lambda_{\delta}^{\tau} W_{\lambda \varrho \varepsilon \zeta \eta \tau}(x) .
$$

Proof. By inserting $U(0, \Lambda) U(0, \Lambda)^{-1}$ in the expression (16) and by using Eqs. (7), (9) one easily gets

$$
\begin{aligned}
& W_{\mu \nu \alpha \beta \gamma \delta}(\Lambda x-\Lambda y)=\left(\Psi_{0}, U(0, \Lambda) U(0, \Lambda)^{-1} h_{\mu \nu}(\Lambda x) U(0, \Lambda)\right. \\
& \left.U(0, \Lambda)^{-1} R_{\alpha \beta \gamma \delta}(\Lambda y) U(0, \Lambda) U(0, \Lambda)^{-1} \Psi_{0}\right) \\
= & \Lambda_{\mu}^{\lambda} \Lambda_{\nu}^{\varrho} \Lambda_{\alpha}^{\varepsilon} \Lambda_{\beta}^{\zeta} \Lambda_{\gamma}^{\eta} \Lambda_{\delta}^{\tau}\left[W_{\lambda \varrho \varepsilon \zeta \eta \tau}(x-y)\right. \\
+ & \left.\partial_{\lambda}\left(\Psi_{0}, \mathscr{F}_{\varrho}(x ; \Lambda) R_{\varepsilon \zeta \eta \tau}(y) \Psi_{0}\right)+\partial_{\varrho}\left(\Psi_{0}, \mathscr{F}_{\lambda}(x ; \Lambda) R_{\varepsilon \zeta \eta \tau}(y) \Psi_{0}\right)\right] .
\end{aligned}
$$

In order to prove the Lemma, we have to show that the distribution

$$
F_{\varrho \varepsilon \zeta \eta \tau}(x, y) \equiv\left(\Psi_{0}, \mathscr{F}_{\varrho}(x ; \Lambda) R_{\varepsilon \zeta \eta \tau}(y) \Psi_{0}\right)
$$

vanishes.

One may easily show that $F_{\varrho \varepsilon \zeta \eta \tau}(x, y)$ depends only on the variable $\xi=x-y$, as a consequence of Eq. (8), and that $F_{\lambda \varepsilon \zeta \eta \tau}(x-y)$ satisfies WLC as a consequence of Eq. (9) and condition (e).

By using the spectral condition (d) and the above properties one may write [6] $F_{\lambda \varepsilon \xi \eta \tau}(\xi)$ as the boundary value of an analytic function $F_{\lambda \varepsilon \xi \eta \tau}(z)$. WLC implies that $F_{\lambda \varepsilon \zeta \eta \tau}(z)$ is analytic [13] in the extended tube: $\mathscr{T}^{\prime} \equiv\{$ union of all the open sets which may be obtained from the forward tube $\mathscr{T}$ by applying all the transformations of the proper complex Lorentz group $\left.L_{+}(C).\right\}$

To prove that $F_{\varrho \varepsilon \zeta \eta \tau}(z)$ vanishes, first we observe that due to the antisymmetry properties of $R_{\varepsilon \zeta \eta \tau}$ the following equation holds

$$
z^{\varepsilon} z^{\zeta} F_{\varrho \varepsilon \zeta \eta \tau}(z) \equiv z^{\varepsilon} H_{\varrho \varepsilon \eta \tau}=0
$$

where $H_{\varrho \varepsilon \eta \tau} \equiv z^{\zeta} F_{\varrho \varepsilon \zeta \eta \tau}$.

Now $\mathscr{T}^{\prime}$ contains intervals of the form $\left\{z: z^{0}=0, z^{j} \neq 0, z^{i}=0, i \neq j\right\}$. Thus, on those intervals one has

$$
z^{1} H_{\varrho 1 \eta \tau}=0, \quad z^{2} H_{\varrho 2 \eta \tau}=0, \quad z^{3} H_{\varrho 3 \eta \tau}=0
$$

and consequently

$$
H_{\text {ei } \tau}=0 \quad i=1,2,3 \quad \text { on } \quad \mathscr{T}^{\prime} .
$$

Thus, $z^{\varepsilon} H_{\varrho \varepsilon \eta \tau}=0$ implies that also $H_{\varrho 0 \eta \tau}$ vanishes on $\mathscr{T}^{\prime}$. In an analogous way one proves that also $F_{\varrho \varepsilon \zeta \eta \tau}$ vanishes on $\mathscr{T}^{\prime}$. 
Lemma 2. The two point function $W_{\mu \nu \alpha \beta \gamma \delta}$ may be written in the following form:

$$
\begin{aligned}
& W_{\mu \nu \alpha \beta \gamma \delta}(x)=\left(g_{\alpha \gamma} g_{\beta \delta}-g_{\alpha \delta} g_{\beta \gamma}\right)\left(g_{\mu \nu} A(x)+\partial_{\mu} \partial_{v} B(x)\right) \\
+ & \left(g_{\beta \delta} g_{\alpha \varrho} g_{\gamma \sigma}+g_{\alpha \gamma} g_{\beta \varrho} g_{\delta \sigma}-g_{\beta \gamma} g_{\alpha \varrho} g_{\delta \sigma}-g_{\alpha \delta} g_{\beta \varrho} g_{\gamma \sigma}\right) \\
\cdot & {\left[\left(\delta_{\mu}^{\varrho} \delta_{v}^{\sigma}+\delta_{\mu}^{\sigma} \delta_{v}^{\varrho}\right) C(x)+\left(\delta_{\mu}^{\varrho} \partial_{\nu} \partial^{\sigma}+\delta_{\nu}^{\varrho} \partial_{\mu} \partial^{\sigma}+\delta_{\mu}^{\sigma} \partial_{\nu} \partial^{\varrho}+\delta_{\nu}^{\sigma} \partial_{\mu} \partial^{\varrho}\right) D(x)\right] } \\
+ & \left(g_{\beta \gamma} \partial_{\alpha} \partial_{\delta}+g_{\alpha \delta} \partial_{\beta} \partial_{\gamma}-g_{\alpha \gamma} \partial_{\beta} \partial_{\delta}-g_{\beta \delta} \partial_{\alpha} \partial_{\gamma}\right)\left[g_{\mu \nu} F(x)+\partial_{\mu} \partial_{v} E(x)\right] \\
+ & \left(g_{\beta \varrho} g_{\gamma \sigma} \partial_{\alpha} \partial_{\gamma}+g_{\alpha \varrho} g_{\delta \sigma} \partial_{\beta} \partial_{\gamma}-g_{\beta \varrho} g_{\delta \sigma} \partial_{\alpha} \partial_{\gamma}-g_{\alpha \varrho} g_{\gamma \sigma} \partial_{\beta} \partial_{\delta}\right)\left(\delta_{\mu}^{\varrho} \delta_{v}^{\sigma}+\delta_{\mu}^{\sigma} \delta_{v}^{\varrho}\right) G(x) \\
+ & {\left[2 \varepsilon_{\varrho \alpha \beta \lambda} \partial^{\lambda}\left(g_{\sigma \gamma} \partial_{\delta}-g_{\sigma \delta} \partial_{\gamma}\right)+2 \varepsilon_{\varrho \gamma \delta \lambda} \partial^{\lambda}\left(g_{\sigma \alpha} \partial_{\beta}-g_{\sigma \beta} \partial_{\alpha}\right)\right.} \\
+ & \varepsilon_{\varrho \alpha \gamma \lambda} \partial^{\lambda}\left(g_{\sigma \beta} \partial_{\delta}-g_{\sigma \delta} \partial_{\beta}\right)+\varepsilon_{\varrho \beta \gamma \lambda} \partial^{\lambda}\left(g_{\sigma \delta} \partial_{\alpha}-g_{\sigma \alpha} \partial_{\delta}\right) \\
+ & \left.\varepsilon_{\varrho \alpha \delta \lambda} \partial^{\lambda}\left(g_{\sigma \gamma} \partial_{\beta}-g_{\sigma \beta} \partial_{\gamma}\right)+\varepsilon_{\varrho \beta \delta \lambda} \partial^{\lambda}\left(g_{\sigma \alpha} \partial_{\gamma}-g_{\sigma \gamma} \partial_{\alpha}\right)\right] \\
& \left(\delta_{\mu}^{\varrho} \delta_{v}^{\sigma}+\delta_{\mu}^{\varrho} \delta_{v}^{\varrho}\right) H(x)
\end{aligned}
$$

where $A, B, C, D, E, F, G, H$ are Lorentz invariant distributions.

Proof. The expression (23) represents the most general tensor with the right symmetry properties

$$
\begin{gathered}
W_{\mu \nu \alpha \beta \gamma \delta}=W_{\nu \mu \alpha \beta \gamma \delta}=-W_{\mu \nu \beta \alpha \gamma \delta}=W_{\mu \nu \beta \alpha \delta \gamma}=W_{\mu \nu \gamma \delta \alpha \beta} \\
W_{\mu \nu \alpha \beta \gamma \delta}+W_{\mu \nu \alpha \gamma \delta \beta}+W_{\mu \nu \alpha \delta \beta \gamma}=0 .
\end{gathered}
$$

The detailed proof is rather lengthy. For details see Ref. [15].

Lemma 3. As a consequence of Bianchi's identities the two point function $W_{\mu \nu \alpha \beta \gamma \delta}$ takes the following form:

$$
\begin{gathered}
W_{\mu \nu \alpha \beta \gamma \delta}(x)=\left(\partial_{\alpha} \partial_{\delta} \delta_{\beta}^{\varrho} \delta_{\gamma}^{\sigma}+\partial_{\beta} \partial_{\gamma} \delta_{\alpha}^{\varrho} \delta_{\delta}^{\sigma}-\partial_{\beta} \partial_{\delta} \delta_{\alpha}^{\varrho} \delta_{\gamma}^{\sigma}-\partial_{\alpha} \partial_{\gamma} \delta_{\beta}^{\varrho} \delta_{\delta}^{\sigma}\right) \\
{\left[g_{\varrho \sigma} g_{\mu \nu}\left(c x^{2}+F\right)+\left(g_{\mu \varrho} g_{\nu \sigma}+g_{\nu \varrho} g_{\mu \sigma}\right)\left(c^{\prime} x^{2}+G\right)+g_{\varrho \sigma} \partial_{\mu} \partial_{\nu} E\right]}
\end{gathered}
$$

where $c$ and $c^{\prime}$ are constants.

Proof. As a first step we shall prove that

$$
H(x)=a x^{2}+b
$$

where $a$ and $b$ are constants. To this purpose we shall use Bianchi's identities, which imply

$$
\varepsilon^{\kappa \alpha \beta \beta} \partial_{\kappa} W_{\mu \nu \alpha \beta \gamma \delta}=0 .
$$

By using the expression (23) for $W_{\mu \nu \alpha \beta \gamma \delta}$, and by contracting Eq. (25) with $g_{v \gamma}$ one gets

$$
\partial_{\kappa}(A+4 C+\square D) \varepsilon^{\mu \delta \kappa \lambda}+10\left(\square g^{\lambda \mu}-\partial^{\lambda} \partial^{\mu}\right) \partial^{\delta} H=0 .
$$


The symmetric and the antisymmetric part with respect to the indices $\mu, \lambda$ must vanish separately. Thus, one has

$$
\begin{aligned}
& A+4 C+\square D=\text { const }, \\
& \left(\square g^{\lambda \mu}-\partial^{\lambda} \partial^{\mu}\right) \partial^{\delta} H=0 .
\end{aligned}
$$

By contracting Eq. (28) with $g_{\lambda \mu}$, one has

$$
\square \partial^{\delta} H=0 .
$$

Hence, Eq. (28) gives

$$
\partial_{\lambda} \partial_{\mu} \partial_{\delta} H=0 \text {. }
$$

The Lorentz invariant solutions of Eq. (29) are the following

$$
H=a x^{2}+b
$$

where $a$ and $b$ are constants. One may easily check that the above expression for $H$ gives zero contribution to the two-point function when substituted in Eq. (23).

By contracting Eq. (25) with $g_{v \lambda}$, one obtains

$$
\partial_{\kappa}(A-2 C) \varepsilon^{\gamma \delta \kappa \mu}=0 \text {, }
$$

i.e.

$$
A=2 C+\text { const . }
$$

Then, Eq. (27) takes the form

$$
6 C+\square D=\text { const . }
$$

Similarly, by contracting Eq. (25) with $\varepsilon^{\gamma \delta \sigma \lambda}$ and by using Eq. (30) and (31) one gets

$$
10\left(\partial^{\sigma} g^{\mu v}+\partial^{v} g^{\mu \sigma}+\partial^{\mu} g^{v \sigma}\right) C+\partial^{\mu} \partial^{v} \partial^{\sigma}(3 B+4 D)=0 .
$$

Multiplying the above Eq. (32) by $\partial_{\mu} g_{\sigma \kappa}-\partial_{\kappa} g_{\sigma \mu}$ yields

$$
\left(\square \delta_{\kappa}^{v}-4 \partial^{v} \partial_{\kappa}\right) C=0 .
$$

As shown in Ref. [10], the Lorentz invariant solutions of Eq. (33) have the following form

$$
C=a_{c} x^{2}+b_{c}
$$

where $a_{c}$ and $b_{c}$ are constants. By substituting the above expression for $C$ in Eq. (32), one may write Eq. (32) in the following way

$$
\partial^{\sigma} \partial^{\mu} \partial^{v}\left(3 B+4 D+\frac{5}{2} a_{c} x^{4}\right)=0 .
$$


This implies

$$
3 B+4 D+\frac{5}{2} a_{c} x^{4}=a_{b} x^{2}+b_{b}
$$

where $a_{b}$ and $b_{b}$ are constants.

On the other hand, by contracting Eq. (25) with $\varepsilon^{\mu \nu \delta \sigma}$ one obtains

i.e.

$$
\left(\square \delta_{\sigma}^{\lambda}-\partial_{\sigma} \partial^{\lambda}\right) \partial^{v}(B-2 D)=0,
$$

$$
B=2 D+a_{d} x^{2}+b_{d}
$$

where $a_{d}$ and $b_{d}$ are constants (Eq. (37) has the same form as Eq. (28)).

Finally, the contraction of Eq. (25) with $\varepsilon^{\mu \gamma \lambda \sigma}$ gives

$$
5\left(g^{v \delta} \partial^{\sigma}+g^{\delta \sigma} \partial^{v}+g^{v \sigma} \partial^{\delta}\right) C+\partial^{\sigma} \partial^{v} \partial^{\delta}(B+3 D)=0 .
$$

When combined with Eqs. (32), (34) and Eq. (38), the above Eq. (39) yields

$$
\begin{aligned}
& B=-\frac{1}{2} a_{c} x^{4}+a x^{2}+b \\
& D=-\frac{1}{4} a_{c} x^{4}+\tilde{a} x^{2}+\tilde{b}
\end{aligned}
$$

where $a, b, \tilde{a}$ and $\tilde{b}$ are constants.

Hence,

$$
\begin{array}{ll}
A=2 a_{c} x^{2}+b_{a}, & B=-\frac{1}{2} a_{c} x^{4}+a x^{2}+b, \\
C=a_{c} x^{2}+b_{c}, & D=-\frac{1}{4} a_{c} x^{4}+\tilde{a} x^{2}+\tilde{b} .
\end{array}
$$

By substituting the above expression for $A, B, C, D$ in (23), after some lengthy algebra one gets

$$
\begin{aligned}
W_{\mu \nu \alpha \beta \gamma \delta}(x)= & \left(\partial_{\alpha} \partial_{\delta} \delta_{\beta}^{\varrho} \delta_{\gamma}^{\sigma}+\partial_{\beta} \partial_{\gamma} \delta_{\alpha}^{\varrho} \delta_{\delta}^{\sigma}-\partial_{\beta} \partial_{\delta} \delta_{\alpha}^{\varrho} \delta_{\gamma}^{\sigma}-\partial_{\alpha} \partial_{\gamma} \delta_{\beta}^{\varrho} \delta_{\delta}^{\sigma}\right) \\
& {\left[g_{\varrho \sigma} g_{\mu \nu}\left(c x^{2}-a_{c} x^{4}+F\right)+\left(g_{\mu \varrho} g_{v \sigma}+g_{v \varrho} g_{\mu \sigma}\left(c^{\prime} x^{2}+G\right)\right.\right.} \\
& +g_{\varrho \sigma} \partial_{\mu} \partial_{v}\left(E+\frac{a_{c}}{24} x^{6}\right)
\end{aligned}
$$

where $c$ and $c^{\prime}$ are suitable constants related to the constants appearing in Eqs. (42), (43). For details see Ref. [15].

Theorem 1. A quantum field theory of Einstein's equations, with the properties $\left.\left.\left.\left.\mathrm{a}^{\prime}\right), \mathrm{b}^{\prime}\right), \mathrm{c}\right), \mathrm{d}\right)$, cannot be weakly local. Otherwise, one has

$$
\left(\Psi_{0}, R_{\lambda \mu \nu \varrho} R_{\alpha \beta \gamma \delta} \Psi_{0}\right)=0 .
$$

Proof. The expression given in Lemma 3 for the two point function $W_{\mu \nu \alpha \beta \gamma \delta}$ has the same form one would get by applying the operator

$$
D_{\alpha \beta \gamma \delta}^{\varrho \sigma}=\partial_{\alpha} \partial_{\delta} \delta_{\beta} \delta_{\gamma}^{\sigma}+\partial_{\beta} \partial_{\gamma} \delta_{\alpha}^{\varrho} \delta_{\delta}^{\sigma}-\partial_{\beta} \partial_{\delta} \delta_{\alpha}^{\varrho} \delta_{\gamma}^{\sigma}-\partial_{\alpha} \partial_{\gamma} \delta_{\beta}^{\varrho} \delta_{\delta}^{\sigma}
$$


to a covariant two point function $W_{\mu \nu \rho \sigma}$ (see Ref. [10]). This is just the operator which gives $R_{\alpha \beta \gamma \delta}$ when applied to $h_{\varrho \sigma}$, see Eq. (6). Thus, one may retrace step by step the proof of the theorem given in Ref. [10] to get Eq. (44).

As a matter of fact, the theorem proved gives restrictions on the invariant functions which appear in the covariant distribution

$$
W_{\mu \nu \alpha \beta \gamma \delta}(x) \equiv D_{\alpha \beta \gamma \delta}^{\varrho \sigma} W_{\mu \nu \varrho \sigma}^{\text {cov. }} .
$$

As the form of $W_{\mu \nu \alpha \beta \gamma \delta}$ as given by Lemma 3 coincides with the form of $W_{\mu \nu \alpha \beta \nu \delta}$ as given in Ref. [10] the same conclusions of Ref. [10] hold in this case.

\section{Discussion of the Results}

It is already clear from the content of Theorem 1 that one cannot hope to quantize the Einstein's equations, while preserving microscopic causality. The argument can be made stronger if one assumes that the metric operator is positive definite on the physical states. Here and in the following by physical states we mean the set $D_{0}$ of vectors which can be obtained from the vacuum state by applying polynomials in the smeared fields $R_{\alpha \beta \gamma \delta}\left(f^{\alpha \beta \gamma \delta}\right)$. Then, one has the following

Corollary 1. If the metric operator $\eta$ is nonnegative in $D_{0}$, then Eq. (44) implies that all the Wightman functions vanish:

$$
\left(\Psi_{0}, R_{\alpha_{1} \beta_{1} \gamma_{1} \delta_{1}}\left(x_{1}\right) \ldots R_{\alpha_{n} \beta_{n} \gamma_{n} \delta_{n}}\left(x_{n}\right) \Psi_{0}\right)=0
$$

and therefore the theory is trivial.

In order to prove it we state beforehand the following

Lemma 4. Given a Hilbert space $H$ in which $\eta=\eta^{+}$, let $H^{\prime}$ be a linear set of vectors such that

$$
\langle\varrho, \eta \varrho\rangle \geqq 0 \quad \forall \varrho \in H^{\prime} .
$$

Let $\psi$ be a vector of $H^{\prime}$ with the property

$$
\langle\psi, \eta \psi\rangle=0 .
$$

Then we have

$$
\langle\phi, \eta \psi\rangle=0 \quad \forall \phi \in H^{\prime} .
$$

Proof. By putting $\varrho=\lambda \phi+\psi, \lambda \in \mathbb{R}, \phi \in H^{\prime}$ in Eq. (46), one finds

$$
0 \leqq\langle\lambda \phi+\psi, \eta(\lambda \phi+\psi)\rangle=\lambda^{2}\langle\phi, \eta \phi\rangle+2 \lambda \operatorname{Re}\langle\psi, \eta \phi\rangle .
$$


As a consequence one has

$$
\operatorname{Re}\langle\psi, \eta \phi\rangle=0 .
$$

On the other hand, by choosing $\varrho^{\prime}=\lambda \phi+i \psi$, one finds

$$
0 \leqq \lambda^{2}\langle\phi, \eta \phi\rangle-2 \lambda \operatorname{Im}\langle\psi, \eta \phi\rangle
$$

from which it follows

$$
\operatorname{Im}\langle\psi, \eta \phi\rangle=0 .
$$

In conclusion we have

$$
\langle\phi, \eta \psi\rangle=0 .
$$

With the aid of this lemma we can now prove Corollary 1 in the following way.

Since $R(f) \equiv R_{\lambda \mu \nu \varrho}\left(f^{\lambda \mu \nu \rho}\right)$ is observable, it must be hermitian with respect to the metric operator $\eta$

Then Eq. (44) implies

$$
R^{+} \eta=\eta R .
$$

$$
\left\langle\eta R(f) \Psi_{0}, R(f) \Psi_{0}\right\rangle=\left\langle\eta \Psi_{0}, R(f) R(f) \Psi_{0}\right\rangle=0 .
$$

Let us now consider the following scalar product

$$
\begin{aligned}
& \left\langle\eta \psi_{0}, R\left(f_{1}\right) \ldots R\left(f_{n}\right) \Psi_{0}\right\rangle \\
= & \left\langle\Psi_{0}, R^{+}\left(f_{1}\right) \ldots R^{+}\left(f_{n-1}\right) \eta R\left(f_{n}\right) \Psi_{0}\right\rangle \\
= & \left\langle R\left(f_{n-1}\right) \ldots R\left(f_{1}\right) \psi_{0}, \eta R\left(f_{n}\right) \Psi_{0}\right\rangle .
\end{aligned}
$$

Since $R\left(f_{n-1}\right) \ldots R\left(f_{1}\right) \Psi_{0} \in D_{0}$ and $\left\langle R\left(f_{n}\right) \Psi_{0}, \eta R\left(f_{n}\right) \Psi_{0}\right\rangle=0$, Lemma 4 implies

$$
\left\langle\eta \Psi_{0}, R\left(f_{1}\right) \ldots R\left(f_{n}\right) \Psi_{0}\right\rangle=0
$$

and, by the nuclear theorem

$$
W_{\alpha_{1} \ldots \delta_{n}}^{(n) . .}\left(x_{1} \ldots x_{n}\right)=\left(\Psi_{0}, R_{\alpha_{1} \beta_{1} \gamma_{1} \delta_{1}}\left(x_{1}\right) \ldots R_{\alpha_{n} \beta_{n} \gamma_{n} \delta_{n}}\left(x_{n}\right) \Psi_{0}\right)=0 .
$$

The above results have been obtained under fairly general assumptions. This proves that many of the solutions suggested in the literature for the problem of quantizing the Einstein's equations are inconsistent with the locality postulate [16].

In particular, any quantum field theory which satisfies assumptions $\left.\left.\left.a^{\prime}\right), b^{\prime}\right), c\right)$ and d), in which the Einstein's equations

$$
\begin{gathered}
R_{\mu \nu} \Psi_{0}=0 \quad R \Psi_{0}=0, \\
\varepsilon^{\mu \nu \varrho \sigma} \partial_{\nu} R_{\alpha \beta \varrho \sigma} \Psi_{0}=0
\end{gathered}
$$


are satisfied in the vacuum state, must necessarily involve a gravitational potential $h_{\mu \nu}$ which is non-covariant and non-local. Thus, two of the most important assumptions of axiomatic quantum field theory must be abandoned if one wants the Einstein's equations satisfied on the physical states, i.e. on $D_{0}$.

The above results shed light on the radiation gauge method [11] of quantizing the Einstein's equations. Non-covariance and non-locality are usually regarded as characteristic features of the radiation gauge. As a matter of fact one may reasonably expect that these peculiarities are confined to a specific choice of the gauge and to the peculiar assumptions which enter in the radiation gauge method like, e.g., Fock representation, vanishing of the time like components of the gravitational potential $\left(h_{0 \mu}=0\right)$, particular choice of the phases in the representation of the Poincare group, temperedness of the fields etc. This is not the case, and one cannot hope to get a quantum field theory of Einstein's equation (i.e. in which Eqs. (47) and (48) hold) without violating Lorentz covariance and microscopic causality. In this respect, the radiation gauge looks much more general and important than usually emphasized in the literature.

In conclusion, the only way out of the difficulties related to the noncovariance and non locality, is to abandon Eqs. (47) and (48). As we will discuss in a following paper, this implies that one must formulate the theory in a Hilbert space in which unphysical states must be present, an indefinite metric must be used etc. All this leads essentially to the Gupta formulation.

\section{References}

1. For a review of the difficulties arising in the quantization of the Einstein's equations. See e.g. Kibble, T.W.: High energy physics and elementary particles (International Atomic Energy Agency, Wien 1965).

2. Strocchi, F.: Phys. Rev., D 2, 2334 (1970).

3. Arnowitt, R., Deser, S., Misner, C. W.: Phys. Rev. 113, 745 (1959); 116, 1322 (1959); 117, 1595 (1960). - Weinberg, S.: Phys. Rev. 134, B 882 (1964); 138, B 988 (1965).

4. Landau, L., Lifchitz,E.: Théorie du champ. Editions Mir, Moscou 1966.

5. This point has been stressed by Weinberg, S.: Phys. Rev. 134, B 882 (1964); 138, B 988 (1965).

6. See Wightman, A.S.: Phys. Rev. 101, 860 (1956). For a detailed discussion see: Streater, R., Wightman, A. S.: PCT, spin and statistics and all that. New York: W. A. Benjamin, Inc. 1964 and Wightman, A. S., Gärding, L.: Arch. Fysik 28, 129 (1964).

7. For the time being it is not necessary to specify which kind of distributions $R_{\mu \nu \varrho \sigma}(x)$ are supposed to be. The following results hold true for a large class of distributions including those introduced by A. M. Jaffe [8].

8. Jaffe, A.M.: Phys. Rev. Letters 17, 661 (1966); Phys. Rev. 158, 1454 (1967).

9. Dirac, P. A. M.: Proc. Roy. Soc. (London), 180, 1 (1942). - Pauli, W.: Rev. Mod. Phys. 15, 176 (1943). - Gupta, S. N.: Proc. Phys. Soc. (London) 63, 681 (1950). Bleuler, K. T.: Helv. Phys. Acta 23, 567 (1950). 
10. Strocchi, F.: Phys. Rev. 166, 1302 (1968).

11. Schwinger, J.: Phys. Rev. 130, 1253 (1963). - Arnowitt, R., Deser, S., Misner, C. W.: Phys. Rev. 113, 745 (1959); 116, 1322 (1959); 117, 1595 (1960).

12. Ogievetsky, V.I., Polubarinov,I.V.: Ann. Phys. (N. Y.) 35, 167, 1965. Appendix.

13. Jost, R.: The general theory of quantized fields (Providence R. I., 1965); Streater, R., Wightman, A.S.: PCT spin and statistics and all that. New York: W. A. Benjamin, Inc. 1964.

14. For a discussion about the operator valued distributions for which WLC can be defined see Ref. [8].

15. Bracci,L.: Tesi di Perfezionamento, Scuola Normale Superiore, Pisa.

16. Kraus, K.: Commun. math. Phys. 9, 339 (1968).

L. Bracci

Istituto di Fisica

dell'Università di Pisa

I-56100 Pisa, Italy 